\title{
Distortion Correction using Enhanced Feature Extraction and Classification
}

\author{
Varinderpal Singh \\ Student \\ UIET, Panjab University \\ Chandigarh
}

\author{
Surender Singh Saini \\ Sr. Scientist \\ CSIO
}

\author{
Jaget Singh \\ Assistant Professor \\ UIET, Panjab University \\ Chandigarh
}

\begin{abstract}
With the advance in technology, different types of machines are used to acquire the images. These sensors acquire information and make it in the form of images. Sometime these images are affected by some distortion like barrel and pincushion. This is because theses sensors have a mark focus on either centre or edge. As it focuses on one point so it cannot be removed but can be corrected after acquiring samples. A number of methods have been used to correct this type of distortion. The previous work which we took under consideration was to collect information by extracting some texture features using that feature to classify the image which will provide the correct information at some sort of point. So, there is still need to improve the results because texture feature. But also edge feature and key point information using different algorithms. Then collect information by classifying them using neural classifier which achieves $93 \%$ accuracy.
\end{abstract}

\section{General Terms}

Distortion Correction, classification.

\section{Keywords}

Barrel, Pincushion, Neural, Features.

\section{INTRODUCTION}

The number of Internet-connected cameras is quickly increasing, and a notable amount of them are used in traffic monitoring. At the moment, many of these are used primarily for simply transferring the image to a human operator, and they lack automatic processing. This is mainly because machine vision-based data mining algorithms require manual configuration and maintenance, involving a considerable effort of skilled personnel and, in many cases, measurements and actions taken in the actual real-life scene [1][6].

Distortion is the difference between ideal image coordinate and actual image coordinate. Geometric distortions are further divided into two type barrel and pin cushion distortions. In barrel image magnification decreases with distance from optical axes. The apparent effect of image has been mapped around a sphere. This distortion is removed by fish eye lens. It takes hemispherical view. It occurs in wide angel lenses.

Image is squeezed into smaller spaces hence appears small as distance between image and camera increases. Pincushion distortions are those in which magnitude increases with distance from optical axis. Lines do not go through the center of the image. These distortions occur in telephoto lenses. In pincushion distortion, corners of squares form elongated points, as in a cushion. Corrective lens elements are used to reduce this fault. This can be removed by different analytical and neural networks.

\section{LITERATURE SURVEY}

Byoung-Kwang Kim et al. (2010) in the paper "Correcting Radial Lens Distortion with Advanced Outlier Elimination" proposed a novel algorithm which extends the concept of outlier to include lines which can cause errors in the estimate of the parameter even though the lines are straight in the three dimensional. The given algorithm can pull out outliers effectively. Hence the given algorithm will determine the distortion parameter correctly than existing algorithms.

Yeonsoo Kim et al. (2010) in the paper "Camera Lens Radial Distortion Compensation by Illuminating the Epipolar Lines with a Projector" propose an automatic radial distortion correction without a manual setting of camras. When the projector takes the straight lines with epipolar ones, the straight lines are not corrected, despite the changes in the shape $\&$ the position between projector $\&$ camera. Therefore, they can utilise the correction parameters by reducing the distance between the extracted lines in the image. In the distorteddd image RMS error is reduced and lines become straight lines.

M. Liedlgruber, A. Uhl, A. V'ecsei et al. (2011) "Statistical analysis of the impact of distortion on an automated classification of celiac disease" In this paper different types of texture feature classifications are given such as, WT-DWT, WT-BBC, WT-LDB etc. These methods convert an image from spatial domain to wavelets (having both frequency and time coordinates). Nearest neighbour classifier is used for feature extraction.

Chien-Chuan Huang et al. (2012) in the paper "A LowComplexity Scaling Scheme for Barrel Distortion Correction" presents a less-complexity scaling method used for pincushion distortion correction. The wide-angle are view of cameras used in applications like surveillance. Images taken by these lens having barrel distortion it means the outer part of the image is supresseded more than the inner. Because to the resolution of the image vary from the display of a target display, scaling techniques are also becoming a major issue. Hence, a low-complexity scaling method presented in this paper.

Luo Shuhua et al. (2013) in the paper "GPU-based Barrel Distortion Correction for Acceleration", which gives two stages one is to take coordinates matching from distorted image to corrected one \& othe is bilinear interpolation i.e. adjoining the points made in the two dimensional lines. It requires a little measure. As the processing speed of on the CPU is slower as that of graphics processing unit (GPU) having fast for data measure processsing. So it can give radial correction accuracy. This paper gives an applicable idea of geometric correction on GPU. GPU is 190 times faster than that of CPU.

Ligang Zhang, Dian Vinod Chandran \& Tjondronegoro in the title of the paper, "Discovering the best feature extraction and selection algorithms for spontaneous facial exression recognition," This paper purposes the different features such 
as texture and geometric features can be extracted from an image, such as SIFT feature.

Dr.S.Vijayarani, Mrs.M.Vinupriya, in the title of "Performance Analysis of Canny and Sobel Edgec Detection Algorithms in Image Mining," The comparative analysis is done between sobel \& canny edge detection has done in which the accuracy of sobel is seventy five percent and as that of canny is $87.5 \%$.

\section{PROPOSED MODEL}

With the advance in technology, different types of machines are used to acquire the images. These sensors acquire information and make it in the form of images. Sometime these images are affected by some distortion like barrel and pincushion. This is because theses sensors have a mark focus on either centre or edge. As it focuses on one point so it cannot be removed but can be corrected after acquiring samples. A number of methods have been used to correct this type of distortion. The previous work which we took under consideration was to collect information by extracting some texture features using that feature to classify the image which will provide the correct information at some sort of point. So, there is still need to improve the results because texture feature. But also edge feature and key point information using different algorithms. Then collect information by classifying them using neural classifier to achieve the following objectives:

- To acquire sample of Chess Board image.

- To decompose image sample by using wavelet.

- To implement feature extraction algorithm to extract texture, edges and key points.

- $\quad$ To calculate accuracy using neural classifier (feed forward neural network).

\subsection{Basic Design of the System}

This proposed work has been implemented in MATLAB using a sample of chess board. The basic design of the system to improve its quality is as shown.

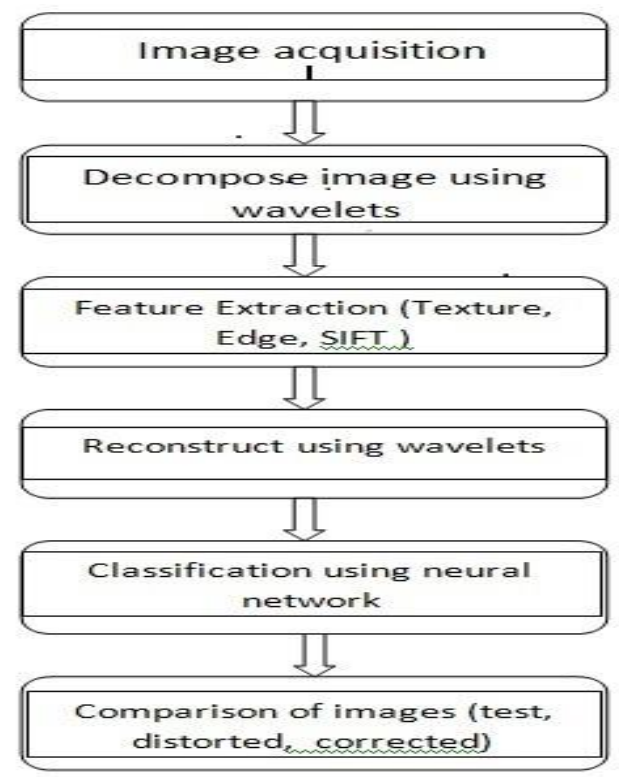

Fig 1: Basic design of the system

\section{IMPLEMENTATION AND SIMULATION RESULTS}

\subsection{Image Acquisition}

The first step is to acquire samples from different datasets to perform the analysis for further processing. A sample of chess board image has been used in this work. After selecting sample image, barrel, pincushion and mixed type of noise has been added to the sample shown in fig 2

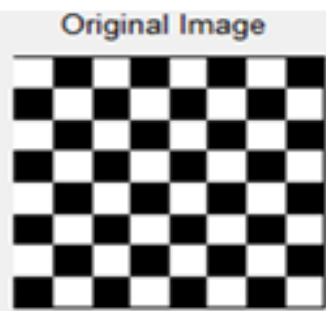

Barrel-Distortion

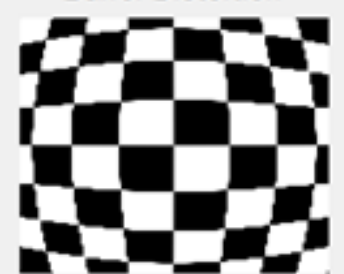

Fig 2: (a) Original Image (b) Pincushion Distorted Image (c) Barrel Distorted Image (d) Mixed Distorted Image

\subsection{Wavelet Decomposition}

Decomposition of image into wavelets is used to convert spatial domain to frequency and time domain. There are different wavelet decomposition techniques used shown in fig 3. Haar wavelet is used as it does better wavelet decomposition. A Haar wavelet is the simplest type of

wavelet. In discrete form, Haar wavelets are related to a mathematical operation called the Haar transform. The Haar transform serves as a prototype for all other wavelet transforms.

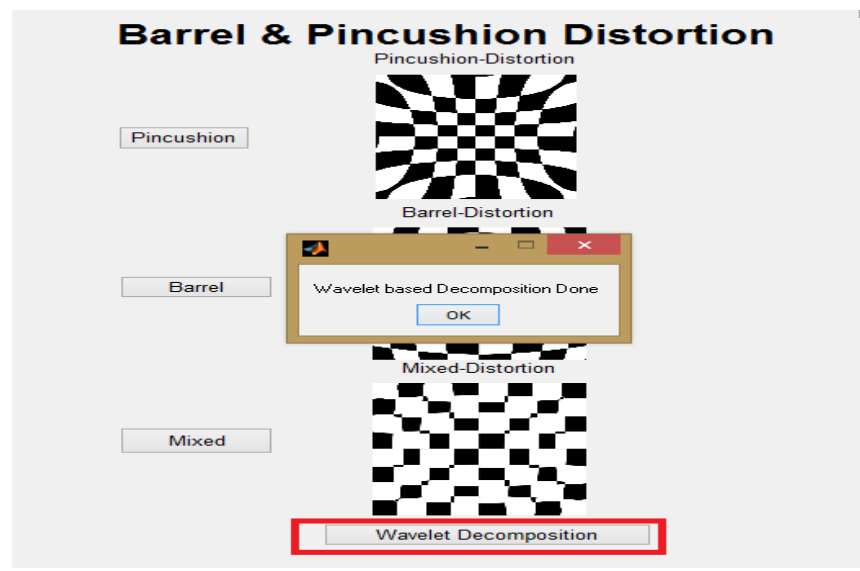

Fig 3: Wavelet Decomposition

\subsection{Feature Extraction}

In this work after decomposing image, different features have been extracted using different methods. Three features are used here that are Texture, Key points and Edge feature. 


\subsubsection{Texture Features}

There is no mathematical definition of texture. Complex visual patterns, composed of spatially organized entities that have characteristic brightness, colour, shape, size. These local subpatterns are characterized by given coarseness, fineness, directionality etc as shown in fig 4 . Texture is homogeneous for human visual system.

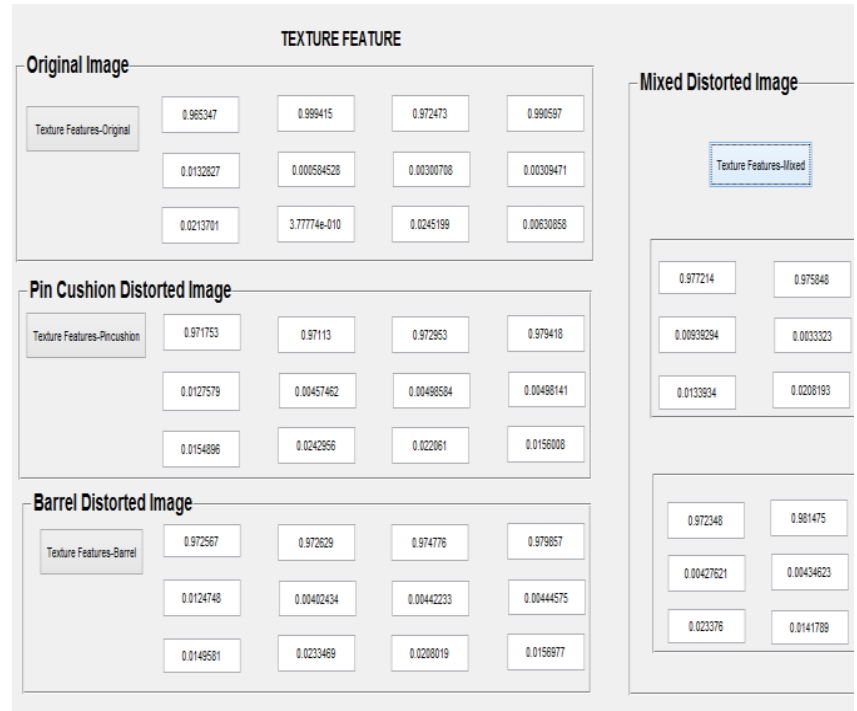

Fig 4: Texture Features

\subsubsection{Keypoints}

Keypoints are extracted using SIFT algorithm. SIFT is Scale Invariant Feature transform algorithm used for feature extraction which mainly focus on the ROI as shown in fig 5 .

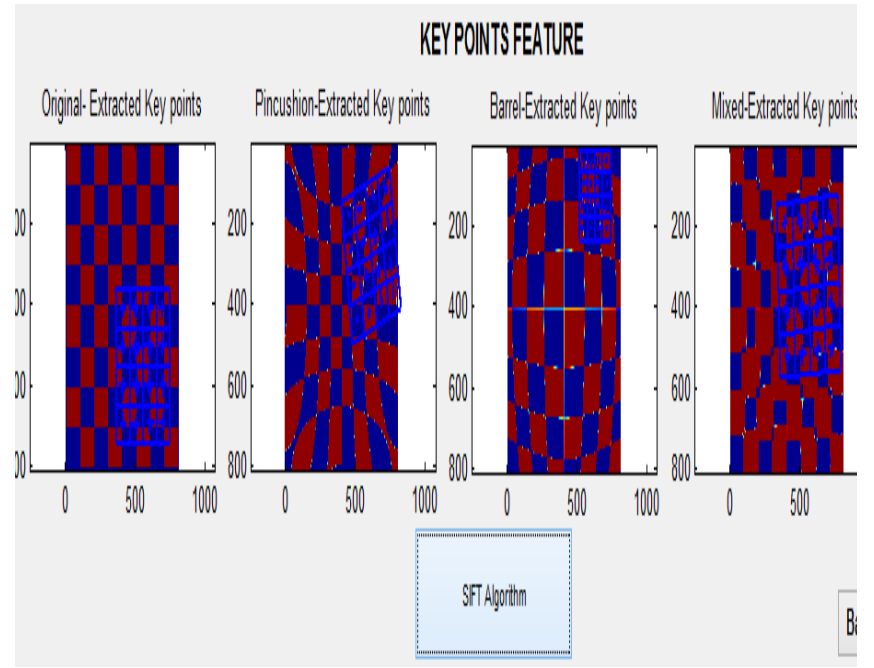

Fig 5: Key Point

\subsubsection{Edge Features:}

The points at which image brightness changes sharply are typically organized into a set of curved line segments termed edges. The algorithms are used in edge detection are:

- Canny Edge detection

- $\quad$ Sobel Edge detection

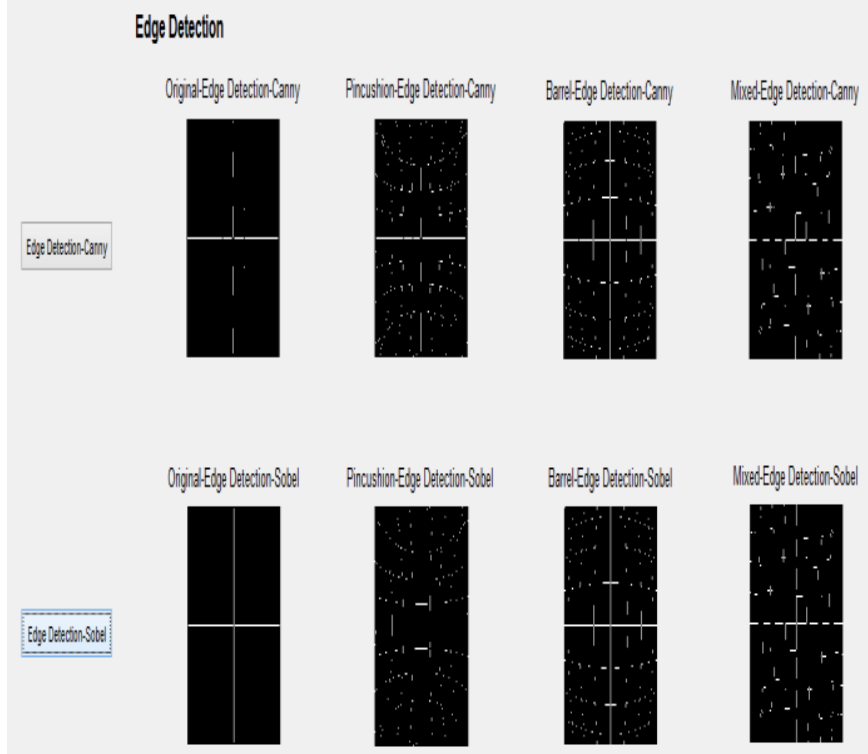

Fig 6: Edge Features

\section{4 . Image Reconstruction}

After extracting all the features from a sample image it can be constructed using wavelet so that the detailed information can be bounded into one single unit. Image reconstruction processhas been shown in fig 7

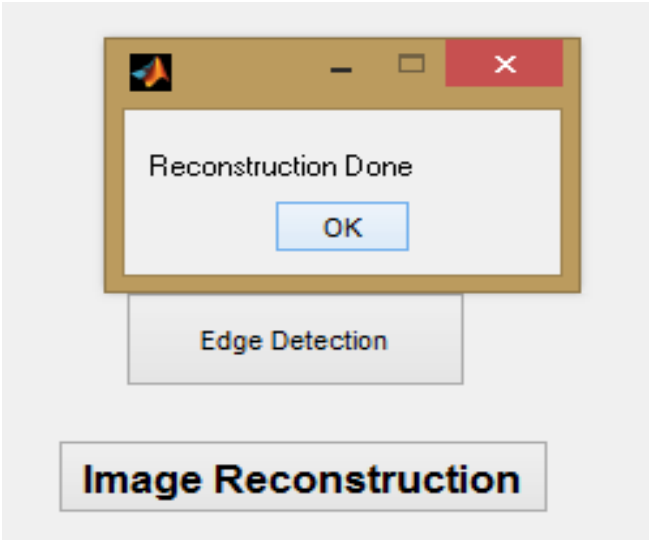

Fig 7: Image Reconstruction

\subsection{Classification}

Results have been classified using neural network where levenberg-arquardt function has been used for training. This achieves $93 \%$ accuracy. 


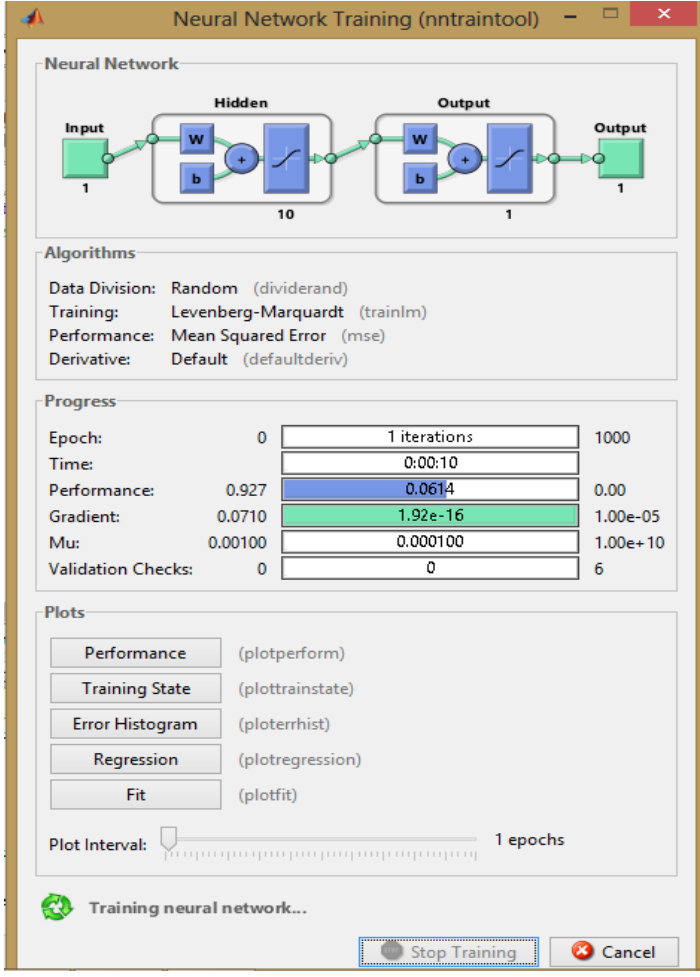

Fig 8: Neural Classification

Distortion correction results are as shown in fig 9.

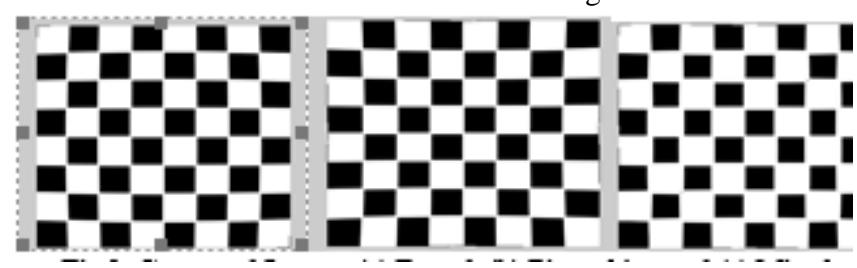

Fig 9: Corrected Images (a) Barrel, (b) Pincushion and (c) Mixed

\section{CONCLUSION}

In this paper, the main focus is to analyze the image features and based on that distortion can be corrected. Various feature extraction methods are introduced in previous researches. Most of the methods either used regional information or boundary information. In this texture features have been extracted which gives the regional details while edge detection gives boundary details. Here SIFT algorithm is used for keypoint detection which gives ROI. On the basis of the feature image can classified and gives approx 93\% accuracy. This work or approach is tested only on chess board image samples. These methods in future used for other images for distortion correction.

\section{REFERENCES}

[1] Byoung-Kwang Kim, Soon-Wook Chung, Moon-Kyu Song, Woo-Jin Song "Correcting Radial Lens Distortion with Advanced Outlier Elimination", Domestic Journal, 2010.
[2] T. Thormählen and H. Broszio, "Automatic line-based estimation of radial lens distortion," Integrated ComputerAided Engineering, vol. 12, no. 2, pp.177-190, 2005.

[3] R. Carroll, M. Agrawala and A. Agarwala, "Optimizing Content-Preserving Projections for Wide-Angle Images," ACM transactions on Graphics, vol. 28, no. 3, article 43, Aug. 2009.

[4] A. Wang, T. Qiu and L. Shao, "A Simple Method of Radial Distortion Correction with Centre of Distortion Estimation," Journal of Mathematical Imaging and Vision, vol. 35, no. 3, pp. 165-172, Jul. 2009.

[5] A. Nowakowski, and W. Skarbek, "Lens Radial Distortion Calibration Using Homography of Central Points," EUROCON 2007. The International Conference "Computer as a Tool”, pp. 340-343, Sep. 2007.

[6] Ricolfe-Viala C, Sanchez-Salmeron A J, Valera A., "Efficient lens distortion correction for decoupling in calibration of wide angle lens cameras", IEEE Sensors Journal, 2013. vol.13, no.2, pp.854-863.

[7] Lee T Y, Wei C H, Lai S H, et al., "Real-time correction of wide-angle lens distortion for images with GPU computing", IEEE Asia Pacific Conference on Circuits and Systems, 2012, pp.456-459

[8] Lee T Y, Chang T S, Lai S H, et al., "Wide-angle distortion correction by Hough transform and gradient estimation". IEEE Visual Communications and Image Processing (VCIP), 2011, pp.1-4.

[9] R. Dong, B. Li, and Q.-M. Chen, "An automatic calibration method for PTZ camera in expressway monitoring system," in Proc. World Congr. Comput. Sci. Inf. Eng., 2009, pp. 636-640.

[10] D. Dawson and S. Birchfield, "An energy minimization approach to auto- matic traffic camera calibration," IEEE Trans. Intell. Transp. Syst., vol. 14, no. 3, pp. 10951108, Sep. 2013.

[11] L. Liu, J. Xing, G. Duan, and H. Ai, "Scene transformation for detector adaptation," Pattern Recognit. Lett., vol. 36, pp. 154-160, Jan. 2013.

[12] Y. Zheng and S. Peng, "A practical roadside camera calibration method based on least squares optimization," IEEE Trans. Intell. Transp. Syst., vol. 15, no. 2, pp. 831843, Apr. 2014

[13] N. K. Kanhere, S. T. Birchfield, and W. A. Sarasua, "Automatic cam- era calibration using pattern detection for vision-based speed sensing," J. Transp. Res. Board, vol. 2086, no. 1, pp. 30-39, 2008.

[14] Z. Zhang, T. Tan, K. Huang, and Y. Wang, "Practical camera calibration from moving objects for traffic scene surveillance," IEEE Trans. Circuits Syst. Video Technol., vol. 23, no. 3, pp. 518-533, Mar. 2013. 